\title{
Collapse and revival of quantum coherence for a harmonic oscillator interacting with a classical fluctuating environment
}

\author{
Jacopo Trapani and Matteo Bina \\ Dipartimento di Fisica, Università degli Studi di Milano, I-20133 Milano, Italy
}

Sabrina Maniscalco

Department of Physics and Astronomy, Turku Centre for Quantum Physics, University of Turku, FI-20014 Turun yliopisto, Finland

\author{
Matteo G. A. Paris* \\ Dipartimento di Fisica, Università degli Studi di Milano, I-20133 Milano, Italia \\ and CNISM, UdR Milano, I-20133 Milano, Italy
}

(Received 4 November 2014; published 17 February 2015)

\begin{abstract}
We address the dynamics of nonclassicality for a quantum system interacting with a noisy fluctuating environment described by a classical stochastic field. As a paradigmatic example, we consider a harmonic oscillator initially prepared in a maximally nonclassical state, e.g., a Fock number state or a Schrödinger-cat-like state, and then coupled to either a resonant or a nonresonant external field. Stochastic modeling allows us to describe the decoherence dynamics without resorting to approximated quantum master equations and to introduce non-Markovian effects in a controlled way. A detailed comparison among different nonclassicality criteria and a thorough analysis of the decoherence time reveal a rich phenomenology whose main features may be summarized as follows: (i) Classical memory effects increase the survival time of quantum coherence and (ii) a detuning between the natural frequency of the system and the central frequency of the classical field induces revivals of quantum coherence.
\end{abstract}

DOI: 10.1103/PhysRevA.91.022113

PACS number(s): 03.65.Yz, 03.65.Ta, 03.65.Xp

\section{INTRODUCTION}

Environment-induced decoherence is the prevailing explanation for the loss of nonclassicality of an open quantum system, being responsible for the relaxation of the system to a statistical mixture of classical-like states [1-3]. In this framework, a nonzero-temperature environment is usually described in terms of a quantized ensemble of simple physical systems, e.g., harmonic oscillators or spins, spanning a wide frequency range and interacting with the quantum system of interest through a suitable interaction Hamiltonian. A set of approximations, such as Born and Markov approximations, is then exploited to obtain a differential master equation describing the dissipative dynamics of the open quantum system [4-10].

In a Markovian approach, the environment time-correlation functions are assumed to decay instantaneously compared to the typical time scale of the system, i.e., memory effects have no influence on the system dynamics. In this context, a thoroughly studied quantum system is the single-mode quantum harmonic oscillator interacting with a bosonic bath of oscillators. For such an open system, the decoherence time, ruling the transition from the quantum to the classical regime, may be identified by different nonclassicality criteria, which have been widely investigated [11-24] and compared [25]. Extensions to multimode systems [26-29] have been analyzed and the decoherence process has been addressed extensively $[30,31]$. Besides the fundamental interest, the analysis of the quantum-to-classical transition has relevant applications in the field of quantum technology. In fact, the generation and detection of nonclassical states is often a prerequisite to

\footnotetext{
*matteo.paris@fisica.unimi.it
}

generate entanglement and discord for quantum information purposes in all-optical setups [32-37].

The assumption of weak coupling between the system and its environment, i.e., the Born approximation, is valid for a wide class of systems. On the other hand, the Markov assumption is violated in several situations of interest, e.g. in biological, optical, or solid-state systems [38-41], where a more detailed description of the environment, including the spectral structure and the inherent memory effects, is required [42-45]. In this regime, decoherence may be less detrimental and the dynamics may even induce recoherence. For this reason a great deal of attention has been devoted to the study of the corresponding non-Markovian dynamics in different systems ranging from quantum optics to mechanical oscillators and harmonic lattices [46-54]. In addition, there is evidence that non-Markovian open quantum systems [55-59] can be useful for quantum technology [60-62].

There are two main paradigms to describe the dynamics of open quantum systems. On the one hand, as mentioned above, one may look at the system and environment as a single global quantum system whose evolution is governed by an overall unitary operator. Upon tracing out the environment's degrees of freedom, we then obtain the dynamics of the system. On the other hand, we may consider the open quantum system under the action of external random forces, i.e., coupled to a stochastic classical field. Here the partial trace is replaced by the average over the different realizations of the stochastic field. While the system-environment approach is more fundamental in nature, the approximations employed to achieve manageable dynamical equations often preclude a detailed description of the dynamics. Indeed, systems of interest for quantum technology generally interact with complex environments, with many degrees of freedom, and 
a fully quantum description may be challenging or even unfeasible. In these situations, classical stochastic modeling of the environment represents a valid and reliable alternative. In fact, it has been shown that for certain system-environment interactions a classical description can be found that is completely equivalent to the quantum description [63-68]. In addition, there is experimental evidence that many quantum systems of interest interact with classical forms of noise, typically Gaussian noise [69-71].

In this paper we consider the paradigmatic case of a quantum harmonic oscillator coupled to a classical stochastic field (CSF) [72]. Here the advantage of choosing a CSF description for the environment is twofold: On the one hand, stochastic modeling allows us to describe the decoherence dynamics without resorting to approximated quantum master equations; on the other hand, we may introduce non-Markovian effects in a controlled way. In addition, stochastic modeling allows us to perform a detailed analysis for relevant kinds of noise, which are usually difficult to describe within a full quantum treatment, e.g., Gaussian noise with and without a Lorentzian spectral density. For qubit systems, description of environment-induced decoherence by the interaction with classical fluctuating field has been successfully carried out [73-82] and this approach has provided insights into the decoherence process for systems of interest in quantum technology.

We will assume that the harmonic oscillator is initially prepared in a maximally nonclassical state, e.g., a Fock number state or a superposition of (possibly mesoscopic) coherent states, the so-called Schrödinger-cat state, and perform a detailed comparison of the decoherence times according to four different criteria for nonclassicality: the nonclassical depth [20], the negativity of the Wigner function [83], the Vogel criterion [17], based on the characteristic function, and the Klyshko criterion for the photon-number distribution [14]. While the sole nonclassical depth criterion represents a proper (i.e., necessary and sufficient) criterion for nonclassicality, the other quantities have the advantage of being good candidates for an experimental implementation.

Our results show that according to all the quantifiers of nonclassicality, the presence of time correlations (i.e., memory effect) in the classical environment enhances the survival time of, say, the Schrödinger-cat state, i.e., it preserves coherence for a longer time compared to the Markovian case. Furthermore, these memory effects become more and more important as long as the central frequency of the stochastic field is detuned with respect to the natural frequency of the harmonic oscillator, up to inducing collapse and revival of quantumness, i.e., collapse and revival of quantum coherence.

The paper is organized as follows. In Sec. II we introduce the system under investigation and the stochastic modeling of the environment, as well as the details of the systemenvironment interaction. We also describe the initial preparation of the system, discuss its nonclassicality, and introduce all the figures of merit used in the subsequent sections. In Sec. III we address in detail the decoherence dynamics of the system interacting with a classical environment described by the Ornstein-Uhlenbeck process. We also evaluate the input-output fidelity of the corresponding quantum channel and discuss its use as a potential indicator of non-Markovian character in our system. In Sec. IV we briefly analyze the decoherence dynamics for an environment described by a CSF with a power-law autocorrelation function. Section V summarizes the paper.

\section{SYSTEM}

We consider a quantum harmonic oscillator interacting with a classical external field. The Hamiltonian of the system may be written as $H=H_{0}+H_{\mathrm{SC}}$, where the free and interaction Hamiltonians are given by

$$
\begin{gathered}
H_{0}=\hbar \omega_{0} a^{\dagger} a, \\
H_{\mathrm{SC}}=\hbar\left[a \bar{B}(t) e^{i \omega t}+a^{\dagger} B(t) e^{-i \omega t}\right],
\end{gathered}
$$

with $\omega_{0}$ the natural frequency of the oscillator and $B(t)$ a time-dependent fluctuating field with central frequency $\omega$ described by a stochastic process with zero mean, whose complex conjugate is $\bar{B}(t)$. Throughout the paper, we will consider the Hamiltonian $H$ rescaled in units of $\hbar \omega_{0}$. As a straightforward consequence, the stochastic classical field $B(t)$, its central frequency $\omega$, and time $t$ become dimensionless quantities (in units of $\omega_{0}$ and $\omega_{0}^{-1}$, respectively).

We assume that the system is initially prepared in a Fock state $|n\rangle$ or in a superposition of coherent states with opposite phases, the so-called Schrödinger-cat state $\left|\psi_{\text {cat }}\right\rangle=$ $\mathcal{N}^{-1 / 2}(|\alpha\rangle+|-\alpha\rangle)$, where $|\alpha\rangle$ indicates a coherent state and the normalization constant is $\mathcal{N}=2\left[1+\exp \left(-2|\alpha|^{2}\right)\right]$. We focus on Fock or Schrödinger-cat states since they have maximal nonclassical depth and thus represent the proper preparation to analyze the quantum-to-classical transition in full detail. Actually, as we will show in the next paragraph, any pure state other than Gaussian pure states would be equally good to address the dynamics of the nonclassical depth. On the other hand, sufficient criteria such as the Vogel criterion and the Klyshko criterion depend on the specific state under investigation and thus having in mind a specific class of states would be helpful to properly address the detection of nonclassicality in realistic conditions.

The nonclassical depth $\eta$ of a quantum state [20] is a quantitative measure of its nonclassicality and is defined as the minimum number of photons to be added to a state in order to erase all of its quantum features. In terms of the $s$-ordered Wigner functions, the nonclassical depth is given by

$$
\eta=\frac{1}{2}(1-\bar{s}),
$$

where $\bar{s}$ is the largest value of $s$ for which the corresponding $s$-ordered Wigner function $W_{s}[\rho](\alpha)$ is positive and may be seen as a classical probability distribution. In turn, we have $0 \leqslant \eta \leqslant 1$. The $s$-ordered characteristic function and the $s$-ordered Wigner function for the Fock states $|n\rangle$ and the Schrödinger-cat states $\left|\psi_{\text {cat }}\right\rangle$, as well as the Schrödingercat-state matrix elements in the Fock basis, are given in the Appendix. As it is apparent from their expressions, the $s$-ordered Wigner functions of both classes of states are not positive functions for any $-1<s \leqslant 1$. Correspondingly, the nonclassical depth $\eta$ of a Fock or Schrödinger-cat state is equal to one [15] independently of $\alpha$ or $n$, i.e., the Schrödinger-cat and the number states are maximally nonclassical states independently of their energy, as the first positive Wigner function corresponds to $s=-1$, i.e., the Husimi $Q$ function. 
More generally, we have that the nonclassical depth is $\eta=1$ [21] for any pure state other than Gaussian pure states (squeezed coherent state); squeezed states have $0 \leqslant \eta \leqslant \frac{1}{2}$ depending on the squeezing parameter, while coherent states have $\eta=0$, properly capturing the fact that they are the closest analog to classical states for the quantum harmonic oscillator.

The Hamiltonian in the interaction picture reduces to

$$
H_{I}(t)=a e^{-i \delta t} \bar{B}(t)+a^{\dagger} e^{i \delta t} B(t),
$$

where $\delta=1-\omega$ is the detuning between the natural frequency of the oscillator and the central frequency of the CSF (in units of $\omega_{0}$ ). The corresponding evolution operator is given by

$$
U(t)=\mathcal{T} \exp \left\{-i \int_{0}^{t} d s H_{I}(s)\right\}
$$

where $\mathcal{T}$ denotes time ordering. Notice, however, that as long as $B\left(t_{1}\right) \bar{B}\left(t_{2}\right)=\left[B\left(t_{1}\right) \bar{B}\left(t_{2}\right)\right]^{*}$, the two-time commutator $\left[H_{I}\left(t_{1}\right), H_{I}\left(t_{2}\right)\right]$ is proportional to the identity

$$
\left[H_{I}\left(t_{1}\right), H_{I}\left(t_{2}\right)\right]=2 i \sin \left[\delta\left(t_{2}-t_{1}\right)\right] B\left(t_{1}\right) \bar{B}\left(t_{2}\right) \mathbb{I}
$$

and this form allows us to evaluate time ordering using the Magnus expansion [84,85], which is exact already at second order. According to the Magnus expansion, the evolution operator may be written as

$$
U(t)=\exp \left(\Omega_{1}+\Omega_{2}\right),
$$

where

$$
\begin{gathered}
\Omega_{1}=-i \int_{0}^{t} d s_{1} H_{I}\left(s_{1}\right)=a^{\dagger} \phi_{t}-a \phi_{t}^{*}, \\
\phi_{t}=-i \int_{0}^{t} d s_{1} e^{i \delta s_{1}} B\left(s_{1}\right),
\end{gathered}
$$

and

$$
\Omega_{2}=\frac{1}{2} \int_{0}^{t} d s_{1} \int_{0}^{s_{1}} d s_{2}\left[H_{I}\left(s_{1}\right), H_{I}\left(s_{2}\right)\right] \propto \mathbb{I} .
$$

Since $\Omega_{2}$ is proportional to the identity, we may write the evolution of an initial density operator $\rho(0)$ as

$$
\rho(t)=\left[e^{\Omega_{1}} \rho(0) e^{\Omega_{1}^{*}}\right]_{B}=\left[D\left(\phi_{t}\right) \rho(0) D^{\dagger}\left(\phi_{t}\right)\right]_{B},
$$

where $D(\mu)=e^{\mu a^{\dagger}-\bar{\mu} a}$ is the displacement operator and $[\cdots]_{B}$ denotes the average over the different realization of the stochastic process. Equation (10) shows that the interaction Hamiltonian with a classical field results in a time-dependent displacement of argument $\phi_{t}$, related to the classical field $B(t)$ and then strongly affected by its stochasticity.

In our system we assume that the CSF $B(t)=B_{x}(t)+$ $i B_{y}(t)$ is described by a Gaussian stochastic process with zero mean $\left[B_{x}(t)\right]_{B}=\left[B_{y}(t)\right]_{B}=0$ and diagonal structure of the autocorrelation matrix

$$
\begin{gathered}
{\left[B_{x}\left(t_{1}\right) B_{x}\left(t_{2}\right)\right]_{B}=\left[B_{y}\left(t_{1}\right) B_{y}\left(t_{2}\right)\right]_{B}=K\left(t_{1}, t_{2}\right),} \\
{\left[B_{x}\left(t_{1}\right) B_{y}\left(t_{2}\right)\right]_{B}=\left[B_{y}\left(t_{1}\right) B_{x}\left(t_{2}\right)\right]_{B}=0,}
\end{gathered}
$$

with the (dimensionless) kernel autocorrelation function $K\left(t_{1}, t_{2}\right)$. Using the Glauber decomposition [13] for the initial state

$$
\rho(0)=\int \frac{d^{2} \mu}{\pi} \chi_{0}[\rho(0)](\mu) D^{\dagger}(\mu),
$$

where the (symmetrically ordered) characteristic function is given by $\chi_{0}[\rho](\mu)=\operatorname{Tr}[\rho D(\mu)]$, we may write the evolved state as

$$
\rho(t)=\int \frac{d^{2} \mu}{\pi}\left[e^{\mu \phi^{*}(t)-\mu^{*} \phi(t)}\right]_{B} \chi_{0}[\rho(0)](\mu) D^{\dagger}(\mu) .
$$

For any Gaussian stationary process, we may write

$$
\left[e^{\mu \phi^{*}(t)-\mu^{*} \phi(t)}\right]_{B}=e^{-|\mu|^{2} \sigma(t)}
$$

and $\sigma(t)$ (following Ref. [86]) can be expressed as

$$
\sigma(t)=\int_{0}^{t} \int_{0}^{t} d s_{1} d s_{2} \cos \left[\delta\left(s_{1}-s_{2}\right)\right] K\left(s_{1}, s_{2}\right) .
$$

The $s$-ordered characteristic function $\chi_{s}[\rho(t)](\mu)$ of the evolved state is given by

$$
\chi_{s}[\rho(t)](\mu)=\chi_{0}[\rho(0)](\mu) e^{\frac{1}{2}|\mu|^{2}[s-2 \sigma(t)]},
$$

which corresponds to a Gaussian noise channel $[11,87]$ :

$$
\rho(t)=G[\rho(0)]=\int \frac{d^{2} \mu}{\pi \sigma(t)} e^{-\frac{|\mu|^{2}}{\sigma(t)}} D(\mu) \rho(0) D^{\dagger}(\mu),
$$

where $\sigma(t)$ in Eq. (16) plays the role of the variance of the Gaussian channel.

In order to obtain quantitative results we focus on the Ornstein-Uhlenbeck (OU) process [72,88], with the autocorrelation function given by

$$
K\left(t_{1}, t_{2}\right)=\frac{1}{2} \lambda \gamma e^{-\gamma\left|t_{1}-t_{2}\right|}
$$

The main conclusions of our analysis, however, are independent of the specific feature of the process, as long as we consider classical fields described by stationary Gaussian processes. In Eq. (19) $\lambda$ is a coupling constant and $\gamma$ is a memory parameter equal to the inverse of the characteristic time of the environment (in units of $\omega_{0}$ ). As we will show in the following sections, the memory effects associated with the interaction with a classical OU field allows the initial state to preserve its nonclassicality for times longer than those achieved with a Markovian environment. As we will see, the smaller $\gamma$ is, the longer the survival time of quantumness at fixed values of the detuning $\delta$. Conversely, for $\gamma \gg 1$, the survival time of the Schrödinger-cat state approaches the Markovian values [25]. Indeed, for $\gamma \gg 1$ the autocorrelation function in Eq. (19) approaches a Dirac $\delta$ function.

For the OU process $\sigma(t)$ may be explicitly written as

$$
\begin{aligned}
\sigma(t)= & \frac{\lambda \gamma}{\left(\gamma^{2}+\delta^{2}\right)^{2}}\left\{\delta^{2}(1+t \gamma)-\gamma^{2}(1-\gamma t)\right. \\
& \left.+e^{-\gamma t}\left[\left(\gamma^{2}-\delta^{2}\right) \cos \delta t-2 \gamma \delta \sin \delta t\right]\right\},
\end{aligned}
$$


leading to the following approximated expressions in some particular regimes:

$$
\begin{gathered}
\sigma(t) \simeq \lambda t+\frac{\lambda}{\gamma} e^{-\gamma t} \cos \delta t, \quad \gamma \gg 1 \\
\sigma(t) \simeq \frac{\lambda \gamma}{\delta^{2}}(1-\cos \delta t), \quad \gamma \ll 1, \delta \gg 1 \\
\sigma(t) \simeq \frac{\lambda \gamma t^{2}}{2}\left(1-\delta^{2} t^{2}\right), \quad \gamma \ll 1, \delta \ll 1 .
\end{gathered}
$$

Overall, the interaction with a classical environment corresponds to a Gaussian channel with the time-dependent width $\sigma(t)$, which fully characterizes the dynamics.

Finally, we notice that the map in Eq. (18) is a solution of the standard Born-Markov quantum optical master equation

$$
\begin{aligned}
\frac{d}{d t} \rho(t)= & \frac{\Gamma}{2}(N+1)\left[2 a \rho(t) a^{\dagger}-a^{\dagger} a \rho(t)-\rho(t) a^{\dagger} a\right] \\
& +\frac{\Gamma}{2} N\left[2 a^{\dagger} \rho(t) a-a a^{\dagger} \rho(t)-\rho(t) a a^{\dagger}\right]
\end{aligned}
$$

in the limits $N \gg 1$ and $\Gamma t \ll 1$, where $N$ is the number of thermal photons in the environment and $\Gamma$ the dissipation rate. Equation (24) describes the open-system dynamics of a harmonic oscillator (weakly) interacting with a Markovian bath of harmonic oscillators at the temperature $\left[\ln \left(1+N^{-1}\right)\right]^{-1}$. In other words, in the regime of high temperature and short times, the interaction with a quantized environment is equivalent to the interaction with a classical stochastic field. The explicit mapping is provided by the relation $\sigma(t) \leftrightarrow \Gamma N t$. Further insight into the meaning of the involved parameters may be gained using a short-time detuning-independent approximation for $\sigma(t) \simeq \frac{1}{2} \lambda \gamma t^{2}$.

\section{DYNAMICS OF QUANTUMNESS}

In this section we address in detail the quantum-toclassical transition, according to four different criteria, for a Schrödinger-cat state and a Fock state interacting with a CSF. We evaluate the decoherence times and analyze whether and how these may increase for a channel with memory, compared to a Markovian one. We also discuss the role of detuning in producing collapse and recoherence effects (collapse and revival of quantumness).

\section{A. Nonclassical depth}

As mentioned above, the nonclassical depth was introduced as the minimum number of thermal photons needed to erase the quantum features of a given state [20]. In the phase space, the nonclassical depth enters as the minimum width of the Gaussian convolution needed to transform the (possibly singular) Glauber $P$ function of a given state into a positive function. According to this measure, Fock number states and Schrödinger-cat states are maximally nonclassical states independent of their energy.

Accordingly, the spirit of the nonclassical depth criterion is to find the smallest interaction time $t_{Q}$ such that the $P$ distribution of the evolved state becomes positive, i.e., the evolved state is a statistical mixture of coherent states. Indeed, the nonclassical depth criterion well captures the intuition of decoherence as relaxation of the system into a statistical mixture of classical states.

As we will see, the interaction with the CSF turns the initial $P$ distribution into a positive function after a finite interaction time $t_{Q}$. In addition, depending on the value of the dimensionless parameters $\lambda, \gamma$, and $\delta$, we may also observe revivals of coherence (revival of quantumness). In order to determine these thresholds, one should consider the evolved state $\rho(t)$ and evaluate the time-dependent value of the nonclassical depth. Actually, it is sufficient to evaluate the nonclassical depth only for the initial state since Eq. (17) shows that the normally ordered characteristic function $\chi_{1}[\rho(t)](\mu)$ (which generates the $P$ distribution) corresponds to the $\tilde{s}$-ordered characteristic function of the initial Schrödinger-cat state $\chi_{\tilde{s}}[\rho(0)](\mu)$, where $\tilde{s}=[1-2 \sigma(t)]$. As the nonclassical depth of the Schrödingercat or Fock state is equal to one, the $P$ distribution becomes positive when it turns into a Husimi $Q$ function, which corresponds to $\tilde{s}=-1$. This happens in a finite (dimensionless) time $t_{Q}$ that is straightforwardly defined by

$$
\sigma\left(t_{Q}\right)=1 \text {. }
$$

For values of $t$ such that $\sigma(t)>1$, the $P$ distribution is a positive function and the state is classical. It is worth noting that the nonclassical depth criterion only depends on $\sigma(t)$, which is independent of the initial-state parameter $\alpha$ or $n$. More generally, for a state with initial nonclassical depth $\eta_{0}$ the decoherence time $t_{Q}$ is given by the solution of the equation $\sigma\left(t_{Q}\right)=\eta_{0}$.

Let us first focus on the resonant interaction $(\delta=0)$. In this case $\sigma(t)$ reduces to

$$
\sigma(t)=\lambda t+\frac{\lambda}{\gamma}\left(e^{-\gamma t}-1\right)
$$

and the equation $\sigma(t)=1$ has a single solution for any pair of values of $\lambda$ and $\gamma$. We thus have collapse of quantumness without any revival. As we anticipated in the previous section, the autocorrelation function of the process approaches a Dirac $\delta$ function in the limit of large $\gamma$. If we perform the limit at this stage we obtain $\lim _{\gamma \rightarrow \infty} \sigma(t)=\lambda t$. This form of $\sigma(t)$ coincides with that obtained using Eq. (24) and assuming that $\lambda=\Gamma N$. In other words, the limit $\gamma \gg 1$ leads to the Markovian regime. This also confirms the idea that $\gamma$ plays the role of a memory parameter. More explicitly, its inverse sets the time for which the field correlations cease to be significative. Large values of $\gamma$ describe environments with no memory of their previous configurations. In the Markovian limit the decoherence time $t_{Q}^{(M)}$ is given by

$$
t_{Q}^{(M)}=\frac{1}{\lambda}=\frac{1}{\Gamma N} .
$$

In the present non-Markovian case, we have

$$
t_{Q}=\frac{\gamma+\lambda}{\gamma \lambda}+\frac{1}{\gamma} \xi\left(-e^{-1-\gamma / \lambda}\right),
$$

where $\xi(x)$ is the product-logarithmic function, i.e., the positive real solution $y$ of the equation $x=y e^{y}$. Using this expression, it is possible to show numerically that $t_{Q}>t_{Q}^{(M)}$ for any value of $\gamma$ and $\lambda$, i.e., the non-Markovian character of the field preserves the initial nonclassicality for longer times compared to the Markovian case. This is illustrated in the 

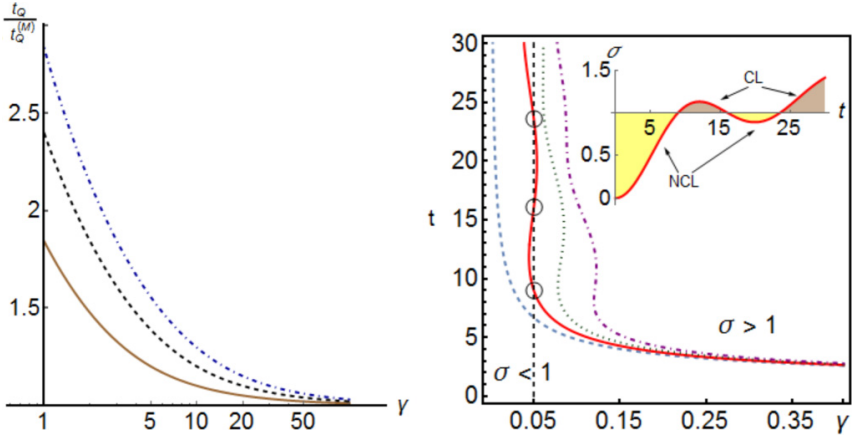

FIG. 1. (Color online) Dynamics of quantumness according to the nonclassical depth criterion. Shown on the left is the dimensionless decoherence time $t_{Q}$ for a resonant interaction as a function of the memory parameter $\gamma$, for different values of the coupling $\lambda=1$ (solid brown curve), $\lambda=2$ (dashed black curve), and $\lambda=3$ (dot-dashed blue curve). For $\gamma \rightarrow \infty, t_{Q}$ approaches the Markovian limit $t_{Q}^{(M)}$ independently of $\lambda$. On the right is a contour plot of $\sigma\left(t_{Q}\right)=1$, in the off-resonance case, as a function of $\gamma$ for a fixed value of the coupling $\lambda=1$ and different values of the detuning $\delta=0.3$ (solid red curve), $\delta=0.4$ (dotted green curve), and $\delta=0.5$ (dot-dashed purple curve). The dashed blue curve is chosen as a reference for the resonant case $\delta=0$. In the regions lying to the left of the curves we have $\sigma(t)<1$, i.e., nonclassicality. The vertical (dashed black) line denotes points at fixed $\gamma=0.05$ and the black circles indicate the three solutions of $\sigma\left(t_{Q}\right)=1$ for $\delta=0.3$. Correspondingly, the regions of nonclassicality (NCL) and classicality (CL) are highlighted in the inset.

left panel of Fig. 1, where we show the ratio $t_{Q} / t_{Q}^{(M)}$ as a function of $\gamma$ for different values of $\lambda$ : The ratio is larger than unity for any value of $\gamma$ and increases for increasing $\lambda$, i.e., nonclassicality is better preserved for larger coupling. For increasing $\gamma$, the decoherence time $t_{Q}$ goes to the Markovian value independently of the value of the coupling.

Let us now analyze what happens if we turn on the detuning between the natural frequency of the system and the central frequency of the field. In this case the equation $\sigma(t)=1$ may have more than one solution (fixing all the parameters $\delta, \gamma$, and $\lambda$ ) and thus revivals of coherence may appear. In the right panel of Fig. 1 we show the contour plot of $\sigma\left(t_{Q}\right)=1$ as a function of time and $\gamma$ for different values of the detuning $\delta$ and for a fixed value $\lambda=1$ of the coupling. The regions lying to the right of the curves correspond to $\sigma(t)>1$, i.e., classicality, whereas regions of nonclassicality $\sigma(t)<1$ lie to the left. There are two main effects: (i) At fixed $\gamma$ the decoherence time $t_{Q}$ increases with the detuning (the effect is more pronounced for smaller $\gamma$ ) and (ii) revivals of quantumness, i.e., collapse followed by revival of quantumness, appear at fixed (and not too large) values of $\gamma$. This is illustrated in the right panel of Fig. 1 and in the corresponding inset, where, for $\delta=0.3$ (solid red line) and $\gamma=0.05, \sigma(t)$ displays recoherence effects. Notice also that for increasing $\gamma$, revivals disappear and $t_{Q}$ becomes more and more independent of the detuning, thus further confirming that for large $\gamma$ we are approaching the Markovian limit.

\section{B. Wigner negativity}

A different notion of nonclassicality is based on the negativity of the Wigner function, which is never singular, but it can take on negative values for nonclassical states, such as Fock states or superposition of coherent states [19]. The notion of nonclassicality arising from the negativity of the Wigner function is not equivalent to the nonclassical depth and it has been linked to nonlocal properties [89,90]. More precisely, it has been shown that positivity of the Wigner function implies that the corresponding quantum state cannot violate any Bell inequality involving only position and momentum measurements. In turn, squeezed vacuum states display a positive Wigner function even though their nonclassical depth ranges from $\eta=0$ to $\eta=\frac{1}{2}$, increasing with energy.

We can evaluate the time $t_{W}$ in which the $P$ function turns into a Wigner function in the very same way we evaluated the nonclassical depth time in the previous section. The condition that $t_{W}$ must satisfy in order to change from a normally ordered into a symmetrically ordered characteristic function is

$$
\sigma\left(t_{W}\right)=\frac{1}{2}
$$

Exactly like the nonclassical depth criterion, the Wigner decoherence time depends only on $\sigma(t)$ and it is not affected by the initial-state parameter $\alpha$ or $n$. For a state with an initial nonclassical depth equal to $\eta_{0}$, the Wigner decoherence time is the solution of $\sigma\left(t_{W}\right)=\eta_{0}-1 / 2$ if $\eta_{0}>\frac{1}{2}$ or $t_{W}=0$ otherwise.

In the Markovian limit $\gamma \gg 1$ the decoherence time $t_{W}^{(M)}$ of the Schrödinger-cat or Fock state is simply half of $t_{Q}^{(M)}$,

$$
t_{W}^{(M)}=\frac{1}{\lambda}=\frac{1}{2 \Gamma N}=\frac{1}{2} t_{Q}^{(M)} .
$$

In the following we investigate whether the interaction with a stochastic field increases the coherence time of the Schrödinger-cat state according to the Wigner negativity criterion and check how the relation in Eq. (29) between $t_{W}$ and $t_{Q}$ is affected by the memory parameter $\gamma$.

The behavior of the Wigner decoherence time is illustrated in Fig. 2. The top left panel shows that $t_{W}$ is significantly increased by the presence of time correlations in the CSF (non-Markovian behavior), whereas the top right panel reveals recoherence effects for certain values of the detuning and memory parameters. In particular, the vertical black line $(\gamma=0.05)$ intercepts the solid red line $(\delta=0.3)$ just once, which means that revivals of nonclassicality displayed in the nonclassical depth criterion (see Fig. 1) are not captured by the Wigner criterion. In the bottom panel of Fig. 2 we compare $t_{Q}$ and $t_{W}$ by showing their ratio as a function of $\gamma$. For large values of the memory parameter $\gamma$ (i.e., in the Markovian limit) the ratio approaches $\frac{1}{2}$, according to Eq. (29). In all the other cases, the ratio increases and approaches the limiting value $\frac{1}{\sqrt{2}}$ for $\gamma \rightarrow 0$. This may be understood as a consequence of the behavior of $\sigma(t)$, as reported in Eq. (21). Indeed, $\sigma(t)$ is basically linear in time for large $\gamma$, whereas it shows a quadratic behavior for $\gamma \ll 1$.

The study of the Wigner negativity criterion in the offresonance regime confirms the main conclusions we drew from the analysis of the nonclassical depth: For $\delta \neq 0$ the Schrödinger-cat-state coherence survives longer and collapse and revival of nonclassicality appear, which is expected by the analogy of the two considered criteria. 

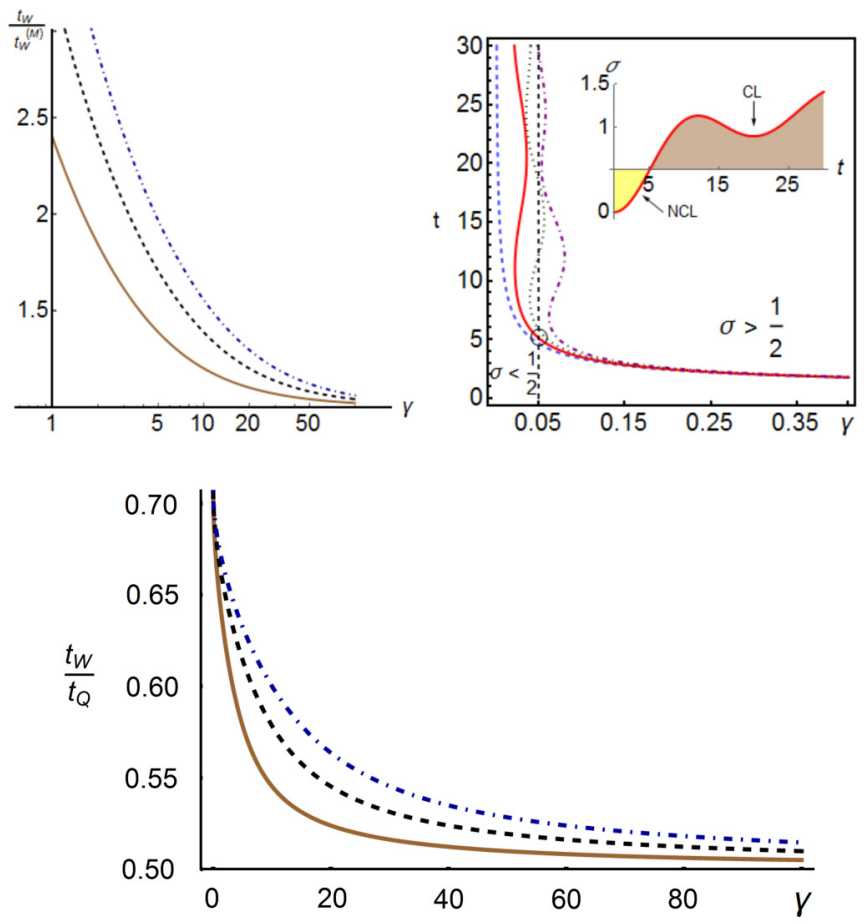

FIG. 2. (Color online) Dynamics of quantumness according to the Wigner negativity criterion. Shown on the top left is the Wigner decoherence time $t_{W}$ for a resonant interaction as a function of the memory parameter $\gamma$, for different values of coupling $\lambda=1$ (solid brown curve), $\lambda=2$ (dashed black curve), and $\lambda=3$ (dot-dashed blue curve). For $\gamma \rightarrow \infty, t_{W}$ approaches the Markovian limit $t_{W}^{(M)}$ independently of $\lambda$. The top right shows a contour plot of $\sigma\left(t_{W}\right)=\frac{1}{2}$, in the off-resonance case, as a function of $\gamma$ for a fixed value of the coupling $\lambda=1$ and different values of the detuning $\delta=0.3$ (solid red curve), $\delta=0.4$ (dotted green curve), and $\delta=0.5$ (dot-dashed purple curve). The dashed blue curve is chosen as a reference for the resonant case $\delta=0$. In the regions lying to the left of the curves we have $\sigma(t)<\frac{1}{2}$, i.e., nonclassicality. The vertical (dashed black) line denotes points at fixed $\gamma=0.05$ and the black circle indicates the solutions of $\sigma\left(t_{W}\right)=\frac{1}{2}$ for $\delta=0.3$. Correspondingly, the regions of nonclassicality (NCL) and classicality (CL) are highlighted in the inset. The bottom plots show the ratio $t_{W} / t_{Q}$ as a function of $\gamma$ with the same values of $\lambda$ as in the top left panel. For $\gamma \gg 1$ the ratio approaches the Markovian value $\frac{1}{2}$, whereas for $\gamma \ll 1$ it approaches $\frac{1}{\sqrt{2}}$, due to the quadratic dependence on time of $\sigma(t)$.

\section{Vogel criterion}

The criteria illustrated in the two previous sections allow us to discriminate classical states from nonclassical ones and to follow the dynamics of decoherence by inspecting the time evolution of a quasiprobability distribution in the phase space. Starting from the criterion based on the positivity of the $P$ function, a sufficient criterion, suitable for experimental implementation, has been suggested and developed [17]. According to this criterion, a state is nonclassical (i.e., its $P$ function is singular) if there exists some complex number $\mu=$ $(u, v)$ such that the normally ordered characteristic function satisfies the inequality

$$
\left|\chi_{1}\left[\rho\left(t_{V}\right)\right](\mu)\right|>1,
$$
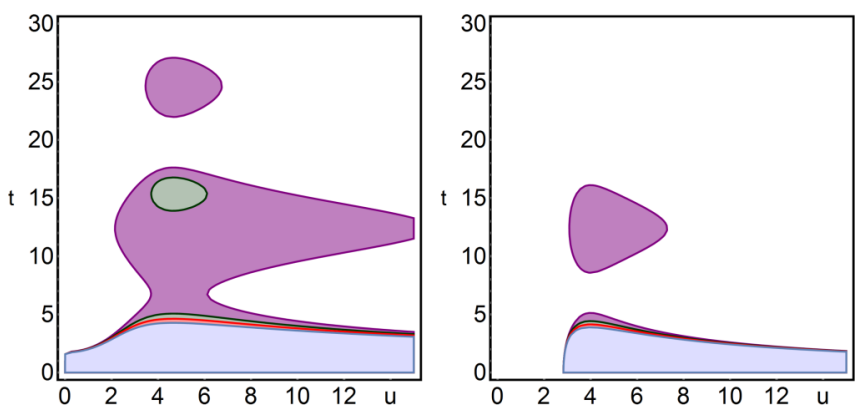

FIG. 3. (Color online) Shown on the left is the Schrödinger-catstate Vogel time $t_{V}$ as a function of $u$, with $|\alpha|=\sqrt{2}$. On the right is the Vogel time $t_{V}$ as a function of $u$ for the Fock state $|2\rangle$. In both panels, $\gamma=0.05$ and filled regions correspond to $\left|\chi_{1}\left[\rho\left(t_{V}\right)\right](u, 0)\right|>$ 1. From bottom to top, the blue region represents the resonant interaction $(\delta=0)$, whereas the red $(\delta=0.3)$, green $(\delta=0.4)$, and purple $(\delta=0.5$ ) regions correspond to the off-resonance case. The spots for the green and the purple regions indicate the presence of revivals of nonclassicality.

where $\chi_{1}[\rho(t)](\mu)=\chi_{0}[\rho(t)](\mu) e^{|\mu|^{2} / 2}$. It should be emphasized that this is only a sufficient condition to characterize nonclassical states. However, it has an advantage stemming from the fact that the symmetric characteristic function can be directly measured via balanced homodyne detection [91].

It is worth noting, however, that in contrast with the two criteria shown previously, the Vogel criterion depends on the state under investigation, i.e., the smallest interaction time $t_{V}$ for which Eq. (30) is satisfied depends on the amplitude $\alpha$ for the Schrödinger-cat state or on the specific Fock state $|n\rangle$. Here we consider Schrödinger-cat states with real amplitude $\alpha=\alpha^{*}=\sqrt{2}$, the reason for this choice being justified later (see Sec. III D). The Fock state $|n=2\rangle$ is chosen such that the number of photons approximates the Schrödinger-cat-state mean number of photons $\left\langle a^{\dagger} a\right\rangle \simeq 2$.

The plots in the left and right panels of Fig. 3, for Schrödinger-cat and Fock states, respectively, show the regions for which $\left|\chi_{1}\left[\rho\left(t_{V}\right)\right](\mu)\right|>1$ as a function of $\operatorname{Re}(\mu)=u$ (with $v=0$ ) and varying detuning parameter $\delta$ (different colors). As it is possible to see in both figures, after a certain time $t_{V}$ nonclassicality disappears, but the revival and collapse of quantumness is present also according to the Vogel criterion (look, for example, at the green and purple regions) and consistently with the two previous criteria, as long as the off-resonance interaction $(\delta \neq 0)$ between the system and the CSF is set.

\section{Klyshko Criterion}

Klyshko introduced a criterion for nonclassicality based on the properties of the photon-number distribution of the state under investigation [14]. The criterion, which is only sufficient for nonclassicality, may be seen as a generalization of the customary condition on the Fano factor of the distribution and states that the state $\rho$ is nonclassical if there exists an integer $n$ such that

$$
B(n)=(n+2) p(n) p(n+2)-(n+1)[p(n+1)]^{2}<0,
$$



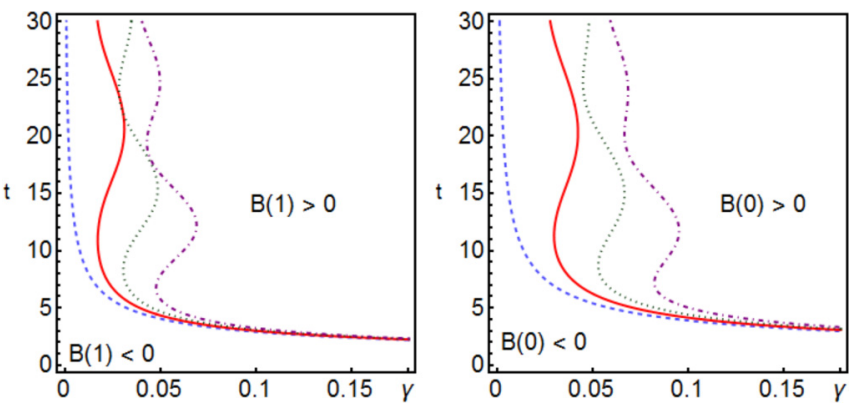

FIG. 4. (Color online) Shown on the left is the decoherence time $t_{K}$ for the Klyshko criterion as a function of $\gamma$ for the Schrödingercat state with $\alpha=\sqrt{2}$. On the right is the decoherence time $t_{K}$ for the Klyshko criterion as a function of $\gamma$ for the Fock state $|2\rangle$. In both panels, the dashed blue curve represents the resonant interaction $(\delta=0)$, whereas solid red $(\delta=0.3)$, dashed green $(\delta=0.4)$, and dot-dashed purple ( $\delta=0.5$ ) curves refer to the off-resonance case. In the regions lying to the left of the curves we have $B(1)<0$ (left) or $B(0)<0$ (right), i.e., nonclassicality.

where $p(n)=\langle n|\rho(t)| n\rangle$ is the photon-number probability of the state $\rho$. Analogously to the Vogel criterion, this nonclassicality evidence is of interest since it is experimentally friendly, being based on the photon distribution, which may be obtained by photon counting or by on-off detectors [92,93].

In our analysis of the Schrödinger-cat-state nonclassicality, according to the Klyshko criterion, we found that $B(1)$ becomes negative after a certain time $t_{K}$ dependent on the detuning $\delta$ and the memory parameter $\gamma$. As it is shown in the left panel of Fig. 4, the Klyshko criterion confirms that the Schrödinger-cat-state survival time increases for short $\gamma$ and it is affected by detuning. Also in this case, collapse and revival of quantumness can be observed, as for fixed $\gamma$ there exists more than one time $t_{K}$ that satisfies the Klyshko criterion (31). A similar behavior is shown for the Fock state $|2\rangle$ in the right panel of Fig. 4, the only difference being the use of the quantity $B(0)$ instead of $B(1)$ to detect the quantum-to-classical transition. As we mentioned earlier, we have chosen $|\alpha|=\sqrt{2}$ for the Schrödinger-cat state. In turn, this choice maximizes the effectiveness of the Klyshko criterion, i.e., is the value corresponding to the longest survival time by the Klyshko criterion [25].

\section{E. A remark about decoherence times}

In the previous sections we went through a quantitative analysis of the nonclassicality dynamics of the Schrödingercat and Fock states, analyzing four different nonclassicality criteria. We described how the interaction of a quantized harmonic oscillator with a CSF, in terms of an OU process, allows us to preserve the nonclassicality of each input state for certain periods of times and this result has been confirmed by the different nonclassicality criteria. In order to emphasize that the Vogel and Klyshko criteria are only sufficient and that they do not show any monotonic properties, a quantitative analysis for both input states is shown in Table I, where we report the times corresponding to the collapse of quantumness achieved according to the four considered criteria, for several values of the detuning $\delta$. In particular, they are obtained by fixing the
TABLE I. Dimensionless decoherence times, obtained for $\gamma=$ $0.05, \lambda=1$, and different values of the detuning $\delta$, corresponding to the collapse of quantumness of the evolved Schrödinger-cat state and the evolved Fock state, according to the four nonclassicality criteria: nonclassical depth $t_{Q}$, Wigner negativity $t_{W}$, Vogel criterion $t_{V}$, and Klyshko criterion $t_{K}$.

\begin{tabular}{lrcrr}
\hline \hline$\delta$ & $t_{Q}$ & $t_{W}$ & $t_{V}$ & \multicolumn{1}{c}{$t_{K}$} \\
\hline \multicolumn{5}{c}{ Schrödinger-cat state } \\
0 & 6.676 & 4.645 & 4.272 & 4.054 \\
0.3 & 8.982 & 5.118 & 4.624 & 4.349 \\
0.4 & 47.467 & 5.823 & 5.067 & 4.694 \\
0.5 & 81.091 & 29.355 & 16.773 & 17.700 \\
\multicolumn{5}{c}{ Fock (number) state } \\
0 & 6.676 & 4.645 & 3.886 & 5.412 \\
0.3 & 8.982 & 5.118 & 4.140 & 6.253 \\
0.4 & 47.467 & 5.823 & 4.425 & 21.329 \\
0.5 & 81.091 & 29.355 & 5.128 & 49.527 \\
\hline \hline
\end{tabular}

value of the parameter $\gamma=0.05$, which is responsible for an appreciable memory effect in the considered OU process.

We note that the times estimated with the Vogel criterion (or the Klyshko criterion) are always shorter than the nonclassical depth and the Wigner negativity decoherence times. This is consistent with the fact that the Vogel and Klyshko criteria provide only sufficient conditions for the loss of quantumness. Indeed, it is possible to still have an amount of nonclassicality in the evolving state that is undetected by these two criteria. Actually, Diósi demonstrated that for some nonclassical states the Vogel criterion is not satisfied [94]. In other words, the evolved Schrödinger-cat or Fock state may still show some quantumness, according to other nonclassicality criteria, while the Vogel criterion is no longer violated. The data in Table I clearly show that it is not possible to establish any order relation between the Vogel and the Klyshko decoherence times and that the two experimentally achievable criteria may fail to return decoherence times comparable to the nonclassical depth time, which should be considered as the proper quantity to individuate the quantum-to-classical transition.

\section{F. Input-output fidelity}

The presence of oscillations in the dynamics of nonclassicality suggests that some form of information backflow from the environment to the system is taking place. This phenomenon is usually associated with quantum non-Markovian character and we want to explore this connection, at least in a qualitative way. In fact, the Markovian character of the quantum map (18) for a coherent input state may be easily proved [58], but Markovian character of coherent states does not necessarily imply Markovian character of Fock states or superposition of coherent states.

The non-Gaussian character of the channel under investigation prevents the analytic evaluation of non-Markovian character measures based on fidelity [58] or Fisher information [57]. On the other hand, since the dynamics induced by the interaction with the CSF is fully described by the quantum channel (18), the input-output fidelity, assessing the dissimilarity between the input state and the output state of a 
quantum map, may be evaluated in a straightforward way as

$$
F_{\mathrm{IO}}=\langle\psi|\mathcal{E}(\rho)| \psi\rangle,
$$

where $\psi$ is the initial state, assumed to be a pure state.

Actually, a nonmonotonic time evolution of $F_{\text {IO }}$ cannot, in general, be interpreted as a signature of backflow of information from the environment to the system (one may construct examples where a system interacts with a Markovian environment and still the IO fidelity oscillates due to some unitary terms in the interaction Hamiltonian). On the other hand, we found that for our system $F_{\text {IO }}$ provides useful information that may be relevant in the qualitative and quantitative characterization of non-Markovian character.

The $F_{\mathrm{IO}}$ for a Schrödinger-cat state and a Fock state interacting with a classical environment are given by

$$
\begin{aligned}
& F_{\mathrm{IO}}^{\text {(cat) }}(t) \\
& =\frac{1+4 e^{2|\alpha|^{2}}+e^{4|\alpha|^{2}}+e^{4|\alpha|^{2} /[1+\sigma(t)]}+e^{4 \sigma(t)|\alpha|^{2} /[1+\sigma(t)]}}{2[1+\sigma(t)]\left[1+e^{2|\alpha|^{2}}\right]^{2}} \\
& F_{\mathrm{IO}}^{(\text {Fock })}(t)=\frac{1}{\sqrt{\pi}[1+\sigma(t)]} \frac{\Gamma\left(n+\frac{1}{2}\right)}{\Gamma(n+1)} \\
& \quad \times{ }_{2} F_{1}\left(-n, \frac{1}{2} ; \frac{1}{2}-n ;\left[\frac{1-\sigma(t)}{1+\sigma(t)}\right]^{2}\right)
\end{aligned}
$$

where ${ }_{2} F_{1}(a, b ; c ; x)$ is a hypergeometric function. The behavior of the IO fidelities in Eqs. (33) and (34) as a function of the interaction time is reported in the top panels of Fig. 5 for $\alpha=\sqrt{2}$ and $n=2$.

For zero detuning, the IO fidelities show a monotonic behavior with the memory parameter $\gamma$, which determines how fast the $F_{\text {IO }}$ decrease. This corresponds to a decoherence dynamics where the state evolves, losing memory of its initial conditions. Conversely, in the presence of detuning the nonresonant curves detach from the respective resonant ones and the $F_{\text {IO }}$ show a nonmonotonic behavior where the state returns to the initial preparation, at least partially. Note that the input-output fidelities of the Schrödinger-cat and Fock states shows a similar behavior, even though they are quantitatively different. In both cases, the oscillating behavior is present for values of $\gamma$ up to a threshold $\gamma^{*}$, which depends on the value of the other parameters $\lambda$ and $\delta$ and do not depend on the initial state, i.e., it represents a property of the channel. For $\lambda=1$ and $\delta=0.3$ (see Fig. 5) we have $\gamma^{*} \simeq 0.082$. Loosely speaking, the existence of the threshold parameter $\gamma^{*}$ means that the evolution is monotonic as long as the time correlations of the environment are weak enough. Remarkably, the same kind of transition may be seen also in the time dependence of the variance $\sigma(t)$. As it is apparent from the bottom panel of Fig. 5, the presence of revivals in the behavior of $\sigma$ also depends on the value of the memory parameter $\gamma$ and it may be proven numerically that also the revivals disappear when $\gamma \gtrsim \gamma^{*}$. Overall, this confirms the backflow of information and the ability of the input-output fidelity to capture this feature of the quantum channel.
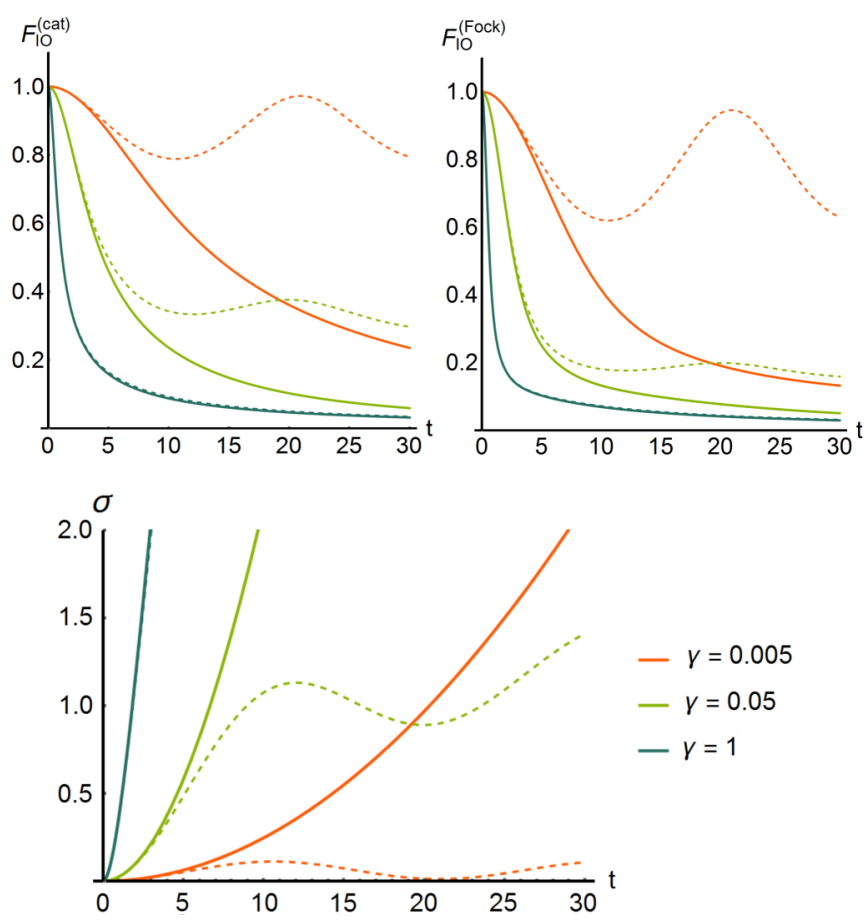

FIG. 5. (Color online) Input-output fidelity as a function of the interaction time for a Schrödinger-cat state with $\alpha=\sqrt{2}$ (top left panel) and a Fock state with $n=2$ (top right panel) for different values of the memory parameter $\gamma$ and the detuning $\delta$ and for fixed coupling $\lambda=1$. Shown for comparison is the behavior of the variance $\sigma$ (bottom panel) in the same conditions. In all the panels the orange curves are for $\gamma=0.005$, green is for $\gamma=0.05$, and blue refers to $\gamma=1$. The solid curves correspond to zero detuning (resonant case) and the dashed ones are for $\delta=0.3$.

\section{POWER-LAW PROCESS}

As mentioned in the Introduction, the main conclusions of our analysis are qualitatively independent of the nature of the CSF used to model the environment. In order to show this explicitly and to briefly illustrate the quantitative effects of a different modeling, we report here the results obtained for a Gaussian process characterized by a long-range power-law autocorrelation function of the form

$$
K\left(t_{1}, t_{2}\right)=\frac{\beta-1}{2} \frac{\gamma \lambda}{\left(1+\gamma\left|t_{1}-t_{2}\right|\right)^{\beta}},
$$

where $\beta>2$. We focus our attention on the nonclassical depth criterion, as it is the most relevant one. The explicit form of $\sigma(t)$ for the power-law process in the case of the resonant interaction $(\delta=0)$ is

$$
\sigma(t)=\lambda t+\lambda \frac{(1+\gamma t)^{2-\beta}-1}{\gamma(\beta-2)} .
$$

This expression can be approximated in some particular regimes to

$$
\begin{gathered}
\sigma(t) \simeq \lambda t+\frac{\lambda \gamma t^{2}}{(\beta-2)(1+\gamma t)^{\beta}} \quad(\gamma \gg 1), \\
\sigma(t) \simeq \frac{\lambda t^{2}}{2}(\beta-1) \quad(\gamma \ll 1) .
\end{gathered}
$$



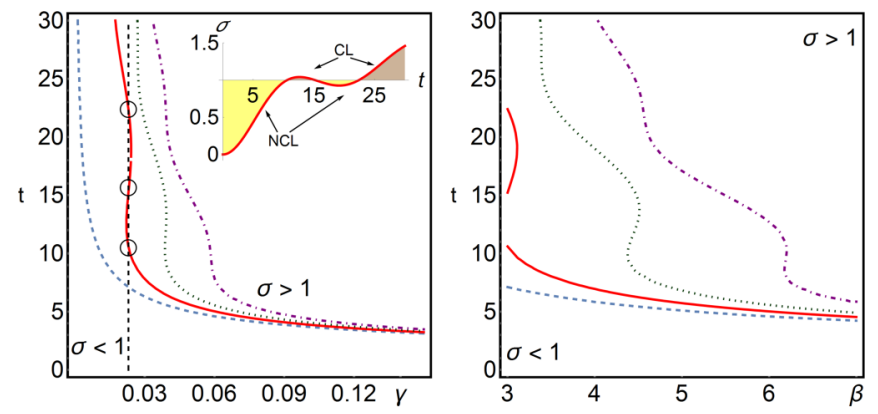

FIG. 6. (Color online) Dynamics of quantumness according to the nonclassical depth criterion for a Schrödinger-cat state evolving in classical environment with a power-law autocorrelation function. In both panels, the dashed blue curve represents the resonant case $\delta=0$, whereas the solid red $(\delta=0.3)$, dotted green $(\delta=0.4)$, dot-dashed purple $(\delta=0.5)$ curves refer to the off-resonance case. Shown on the left is the nonclassical depth time $t_{Q}$ as a function of $\gamma$ in the case of a Gaussian power-law process, for fixed $\beta=3$ and $\lambda=1$. For $\gamma \gg 1$ the nonclassical depth time $t_{Q}$ approaches the Markovian limit independently of $\delta$. Collapse and revival of quantumness are highlighted by the circles along the dashed black line at $\gamma=0.023$ and, correspondingly, in the inset. On the right is the nonclassical depth time $t_{Q}$ as a function of $\beta$ and fixed $\gamma=0.023$.

As we can see from (37), for $\gamma \rightarrow \infty$ the nonclassical depth time approaches the Markovian limit $\sigma(t) \propto t$. Also for the power-law process $\gamma$ plays the role of a memory parameter. In the nonresonant case, the analytic form of $\sigma(t)$ is extremely complex and is not reported in this paper, whereas the results are explained in the following. The presence of collapse and revival of quantumness for the nonresonant interaction is shown for an initial Schrödinger-cat state in the left panel of Fig. 6, where for fixed $\gamma$ and different choices of the detuning parameter $\delta \neq 0$ we can see more than one value of time $t_{Q}$ for which the nonclassical depth criterion is satisfied. In the right panel of Fig. 6 we show the nonclassical depth time as a function of the parameter $\beta$ of the power-law autocorrelation function. Furthermore, the presence of collapse and revival of nonclassicality depends not only on the particular combination of parameters $(\delta, \gamma)$, but also on the parameter $\beta$ itself. Actually, Fig. 6 shows that nonclassicality revivals can be also observed for the power-law process just like for the OU process and that this phenomenon is mostly due to the introduction of the detuning parameter.

\section{CONCLUSION}

In this paper we have investigated the quantum-to-classical transition for a harmonic oscillator initially prepared in a maximally nonclassical state and then interacting with a classical fluctuating field. As a result, we have shown that modeling the environment by means of classical stochastic fields allows us to properly describe the decoherence process in the presence of memory effects, without resorting to approximated quantum master equations. In particular, we have been able to introduce non-Markovian effects in a controlled way, upon describing the phenomenology of the system by the memory parameter $\gamma$ of the CSF. We have also shown that the presence of a Markovian regime of interaction is made possible by using a suitable set of limiting values of the parameters, thus recovering the results of a full quantum treatment.

Our results show that the presence of classical memory in the environment strongly influences the decoherence time, increasing the survival time of nonclassicality and leading to dynamical collapse and revival of quantumness. In particular, when the environment spectrum contains the natural frequency of the oscillator we observe an increase of the survival time compared to the Markovian case, whereas in the presence of a detuning we see the occurrence of collapse and revival of quantumness, as indicated by collapses and revivals of nonclassicality. In order to address this phenomenon quantitatively, we have analyzed the behavior of four different criteria introduced to prove nonclassicality, also relating them to experimentally observable quantities. All these quantifiers agree in describing the nontrivial decoherence process and the revivals of nonclassicality, thus supporting the validity of our model and the main conclusions of our analysis, which may be summarized as follows: (i) Classical memory effects increase the survival time of quantumness and (ii) a detuning between the natural frequency of the system and the central frequency of the environment produces revivals of quantumness.

\section{ACKNOWLEDGMENTS}

This work was supported by MIUR (FIRB "LiCHIS" Grant No. RBFR10YQ3H). M.G.A.P. thanks C. Benedetti, M. G. Genoni, and M. A. C. Rossi for discussions.

\section{APPENDIX}

The $s$-ordered characteristic function of the Schrödinger-cat state $\rho_{\text {cat }}=\left|\psi_{\text {cat }}\right\rangle\left\langle\psi_{\text {cat }}\right|$ is given by

$$
\begin{aligned}
\chi_{s}\left[\rho_{\text {cat }}\right](\mu)= & \frac{2}{\mathcal{N}} e^{-(1-s)|\mu|^{2} / 2}\left[\cos \left(2 \operatorname{Im} \mu \alpha^{*}\right)\right. \\
& \left.+e^{-2|\alpha|^{2}} \cosh \left(2 \operatorname{Re} \mu \alpha^{*}\right)\right] .
\end{aligned}
$$

The $s$-ordered Wigner function of the Schrödinger-cat state is given by

$$
\begin{aligned}
& W_{s}\left[\rho_{\text {cat }}\right](\beta) \\
& =\frac{2 e^{-2 /|\beta|^{2}(1-s)}}{\mathcal{N} \pi(1-s)}\left[\exp \left\{\frac{2 s|\alpha|^{2}}{1-s}\right\} \cos \left(\frac{4}{1-s} \operatorname{Re} \beta \alpha^{*}\right)\right. \\
& \left.\quad+\exp \left\{-\frac{2|\alpha|^{2}}{1-s}\right\} \cosh \left(\frac{4}{1-s} \operatorname{Im} \beta \alpha^{*}\right)\right] .
\end{aligned}
$$

The matrix elements of the Schrödinger-cat state in the Fock basis are given by

$$
\rho_{n, m}=\frac{1}{\mathcal{N}} e^{-|\alpha|^{2}} \frac{\alpha^{n}\left(\alpha^{*}\right)^{m}}{\sqrt{n ! m !}}\left[1+(-1)^{n}\right]\left[1+(-1)^{m}\right] .
$$

The $s$-ordered characteristic function of a generic Fock state $\rho_{F}=|n\rangle\langle n|$ is given by

$$
\chi_{s}\left[\rho_{F}\right](\mu)=\exp \left\{-\frac{(1-s)|\mu|^{2}}{2} L_{n}\left(|\mu|^{2}\right)\right\},
$$


where $L_{n}(x)$ is the Laguerre polynomial of order $n$. The $s$-ordered Wigner function of the Fock state is given by

$$
W_{s}\left[\rho_{F}\right](\beta)=(-1)^{n} \frac{2}{\pi(1-s)}\left(\frac{1+s}{1-s}\right)^{n} \exp \left\{-\frac{2|\beta|^{2}}{1-s}\right\} L_{n}\left(\frac{4|\beta|^{2}}{1-s^{2}}\right) .
$$

[1] S. Haroche and J.-M. Raimond, Exploring the Quantum (Oxford University Press, Oxford, 2006).

[2] W. H. Zurek, Phys. Today 44 (10), 36 (1991).

[3] J. P. Paz, S. Habib, and W. H. Zurek, Phys. Rev. D 47, 488 (1993).

[4] A. Kossakowski, Rep. Math. Phys. 3, 247 (1972).

[5] V. Gorini, A. Kossakowski, and E. C. G. Sudarshan, J. Math. Phys. 17, 821 (1976).

[6] G. Lindblad, Commun. Math. Phys. 48, 119 (1976).

[7] T. Banks, L. Susskind, and M. E. Peskin, Nucl. Phys. B 244, 125 (1984).

[8] H. Carmichael, An Open Systems Approach to Quantum Optics (Springer, Berlin, 1993).

[9] H. P. Breuer and F. Petruccione, The Theory of Open Quantum Systems (Oxford University Press, Oxford, 2002).

[10] U. Weiss, Quantum Dissipative Systems, 3rd ed. (World Scientific, Singapore, 2008).

[11] R. J. Glauber, Phys. Rev. 131, 2766 (1963).

[12] E. C. G. Sudarshan, Phys. Rev. Lett. 10, 277 (1963).

[13] K. E. Cahill and R. J. Glauber, Phys. Rev. 177, 1882 (1969).

[14] D. N. Klyshko, Phys. Lett. A 213, 7 (1996).

[15] M. Takeoka, M. Ban, and M. Sasaki, J. Opt. B 4, 114 (2002).

[16] R. Egger, H. Grabert, and U. Weiss, Phys. Rev. E 55, R3809 (1997).

[17] W. Vogel, Phys. Rev. Lett. 84, 1849 (2000).

[18] Th. Richter and W. Vogel, Phys. Rev. Lett. 89, 283601 (2002).

[19] A. Kenfack and K. Zyczkowski, J. Opt. B 6, 396 (2004).

[20] C. T. Lee, Phys. Rev. A 44, R2775 (1991).

[21] N. Lutkenhaus and S. M. Barnett, Phys. Rev. A 51, 3340 (1995).

[22] J. K. Korbicz, J. I. Cirac, J. Wehr, and M. Lewenstein, Phys. Rev. Lett. 94, 153601 (2005).

[23] J. K. Asbóth, J. Calsamiglia, and H. Ritsch, Phys. Rev. Lett. 94, 173602 (2005).

[24] T. Kiesel, W. Vogel, V. Parigi, A. Zavatta, and M. Bellini, Phys. Rev. A 78, 021804R (2008).

[25] J. Paavola, M. J. W. Hall, M. G. A. Paris, and S. Maniscalco, Phys. Rev. A 84, 012121 (2011).

[26] I. P. Degiovanni, M. Genovese, V. Schettini, M. Bondani, A. Andreoni, and M. G. A. Paris, Phys. Rev. A 79, 063836 (2009).

[27] A. Miranowicz, M. Bartkowiak, X. Wang, Y. X. Liu, and F. Nori, Phys. Rev. A 82, 013824 (2010).

[28] J. S. Ivan, S. Chaturvedi, E. Ercolessi, G. Marmo, G. Morandi, N. Mukunda, and R. Simon, Phys. Rev. A 83, 032118 (2011).

[29] G. Brida, M. Bondani, I. P. Degiovanni, M. Genovese, M. G. A. Paris, I. Ruo Berchera, and V. Schettini, Found. Phys. 41, 305 (2011).

[30] A. Serafini, M. G. A. Paris, F. Illuminati, and S. De Siena, J. Opt. B 7, 19 (2005).

[31] A. Serafini, S. De Siena, F. Illuminati, and M. G. A. Paris, J. Opt. B 6, 591 (2004).

[32] M. G. A. Paris, Phys. Lett. A 225, 28 (1997).

[33] M. G. A. Paris, Phys. Rev. A 62, 033813 (2000).
[34] M. S. Kim, W. Son, V. Buzek, and P. L. Knight, Phys. Rev. A 65, 032323 (2002).

[35] W. Xiang-bin, Phys. Rev. A 66, 024303 (2002).

[36] S. Olivares and M. G. A. Paris, Phys. Rev. Lett. 107, 170505 (2011).

[37] A. Ferraro and M. G. A. Paris, Phys. Rev. Lett 108, 260403 (2012).

[38] S. Maniscalco and F. Petruccione, Phys. Rev. A 73, 012111 (2006).

[39] X.-T. Liang, Phys. Rev. E 82, 051918 (2010).

[40] P. Rebentrost and A. Aspuru-Guzik, J. Chem. Phys. 134, 101103 (2011).

[41] A. Chiuri, C. Greganti, L. Mazzola, M. Paternostro, and P. Mataloni, Sci. Rep. 2, 968 (2012).

[42] B.-H. Liu, L. Li, Y.-F. Huang, C.-F. Li, G.-C. Guo, E.-M. Laine, H.-P. Breuer, J. Piilo, Nat. Phys. 7, 931 (2011).

[43] A. Smirne, D. Brivio, S. Cialdi, B. Vacchini, and M. G. A. Paris, Phys. Rev. A 84, 032112 (2011).

[44] J. Piilo, S. Maniscalco, K. Harkonen, and K.-A. Suominen, Phys. Rev. Lett. 100, 180402 (2008).

[45] A. Smirne, S. Cialdi, G. Anelli, M. G. A. Paris, and B. Vacchini, Phys. Rev. A 88, 012108 (2013).

[46] S. Maniscalco, S. Olivares, and M. G. A. Paris, Phys. Rev. A 75, 062119 (2007).

[47] J. Anders, Phys. Rev. A 77, 062102 (2008).

[48] J. P. Paz and A. J. Roncaglia, Phys. Rev. A 79, 032102 (2009).

[49] R. Vasile, S. Olivares, M. G. A. Paris, and S. Maniscalco, Phys. Rev. A 80, 062324 (2009).

[50] R. Vasile, P. Giorda, S. Olivares, M. G. A. Paris, and S. Maniscalco, Phys. Rev. A 82, 012313 (2010).

[51] F. Galve, G. L. Giorgi, and R. Zambrini, Phys. Rev. A 81, 062117 (2010).

[52] L. A. Correa, A. A. Valido, and D. Alonso, Phys. Rev. A 86, 012110 (2012).

[53] A. Cazzaniga, S. Maniscalco, S. Olivares, and M. G. A. Paris, Phys. Rev. A 88, 032121 (2013).

[54] V. Venkataraman, A. D. K. Plato, T. Tufarelli, and M. S. Kim, J. Phys. B 47, 015501 (2014).

[55] H.-P. Breuer, E.-M. Laine, and J. Piilo, Phys. Rev. Lett. 103, 210401 (2009).

[56] Á. Rivas, S. F. Huelga, and M. B. Plenio, Phys. Rev. Lett. 105, 050403 (2010).

[57] X.-M. Lu, X. Wang, and C. P. Sun, Phys. Rev. A 82, 042103 (2010).

[58] R. Vasile, S. Maniscalco, M. G. A. Paris, H.-P. Breuer, and J. Piilo, Phys. Rev. A 84, 052118 (2011).

[59] D. Chruściński and S. Maniscalco, Phys. Rev. Lett. 112, 120404 (2014).

[60] R. Vasile, S. Olivares, M. G. A. Paris, and S. Maniscalco, Phys. Rev. A 83, 042321 (2011).

[61] A. W. Chin, S. F. Huelga, and M. B. Plenio, Phys. Rev. Lett. 109, 233601 (2012). 
[62] B. Bylicka, D. Chruściński, and S. Maniscalco, Sci. Rep. 4, 5720 (2014).

[63] J. Helm and W. T. Strunz, Phys. Rev. A 80, 042108 (2009).

[64] J. Helm, W. T. Strunz, S. Rietzler, and L. E. Würflinger, Phys. Rev. A 83, 042103 (2011).

[65] D. Crow and R. Joynt, Phys. Rev. A 89, 042123 (2014).

[66] W. M. Witzel, K. Young, and S. Das Sarma, Phys. Rev. B 90, 115431 (2014).

[67] W. T. Strunz, L. Diósi, and N. Gisin, Phys. Rev. Lett. 82, 1801 (1999).

[68] J. T. Stockburger and H. Grabert, Phys. Rev. Lett. 88, 170407 (2002).

[69] O. Astafiev, Yu. A. Pashkin, Y. Nakamura, T. Yamamoto, and J. S. Tsai, Phys. Rev. Lett. 93, 267007 (2004).

[70] Y. M. Galperin, B. L. Altshuler, J. Bergli, and D. V. Shantsev, Phys. Rev. Lett. 96, 097009 (2006).

[71] B. Abel and F. Marquardt, Phys. Rev. B 78, 201302(R) (2008).

[72] C. W. Gardiner, Handbook of Stochastic Methods (Springer, Berlin, 1983).

[73] E. Paladino, L. Faoro, G. Falci, and R. Fazio, Phys. Rev. Lett. 88, 228304 (2002).

[74] L. Cywiński, R. M. Lutchyn, C. P. Nave, and S. Das Sarma, Phys. Rev. B 77, 174509 (2008).

[75] C. Benedetti, F. Buscemi, P. Bordone, and M. G. A. Paris, Int. J. Quantum Inf. 10, 1241005 (2012).

[76] H. J. Wold, H. Brox, Y. M. Galperin, and J. Bergli, Phys. Rev. B 86, 205404 (2012).

[77] C. Benedetti, F. Buscemi, P. Bordone, and M. G. A. Paris, Phys. Rev. A 87, 052328 (2013).
[78] J.-T. Hung, L. Cywiński, X. Hu, and S. Das Sarma, Phys. Rev. B 88, 085314 (2013).

[79] E. Paladino, Y. M. Galperin, G. Falci, and B. L. Altshuler, Rev. Mod. Phys. 86, 361 (2014).

[80] C. Benedetti, M. G. A. Paris, and S. Maniscalco, Phys. Rev. A 89, 012114 (2014).

[81] C. Benedetti and M. G. A. Paris, Int. J. Quantum Inf. 12, 1461004 (2014).

[82] M. A. C. Rossi, C. Benedetti, and M. G. A. Paris, Int. J. Quantum Inf. 13, 1560003 (2015).

[83] R. L. Hudson, Rep. Math. Phys. 6, 249 (1974).

[84] W. Magnus, Commun. Pure Appl. Math. 7, 649 (1954).

[85] S. Blanes, F. Casas, J. A. Oteo, and J. Ros, Phys. Rep. 470, 151 (2008).

[86] R. R. Puri, Mathematical Methods of Quantum Optics (Springer, Berlin, 2001).

[87] A. S. Holevo, Probl. Peredachi Inf. 9, 3 (1973) [Problems of Information Transmission 9, 177 (1973)].

[88] G. E. Uhlenbeck and L. S. Ornstein, Phys. Rev. 36, 823 (1930).

[89] O. Cohen, Phys. Rev. A 56, 3484 (1997).

[90] K. Banaszek and K. Wodkiewicz, Phys. Rev. A 58, 4345 (1998).

[91] A. I. Lvovsky and J. H. Shapiro, Phys. Rev. A 65, 033830 (2002).

[92] A. R. Rossi, S. Olivares, and M. G. A. Paris, Phys. Rev. A 70, 055801 (2004).

[93] G. Zambra, A. Andreoni, M. Bondani, M. Gramegna, M. Genovese, G. Brida, A. Rossi, and M. G. A. Paris, Phys. Rev. Lett. 95, 063602 (2005).

[94] L. Diósi, Phys. Rev. Lett. 85, 2841 (2000). 